\title{
Phytoextraction du cadmium, du cuivre, du plomb et du zinc par Vetiveria nigritana en sols ferrugineux tropicaux et en sols vertiques au Burkina Faso (Afrique de l'ouest)
}

\author{
Issaka SENOU ${ }^{1 *}$, Zacharia GNANKAMBARY ${ }^{2}$, Antoine N. SOME ${ }^{1}$ et \\ Michel P. SEDOGO ${ }^{2}$ \\ ${ }^{1}$ Laboratoire des Systèmes Naturels, des Agro-systèmes et de l'Ingénierie de l'Environnement (Sy.N.A.I.E), \\ Institut du Développement Rural (IDR), Université Polytechnique de Bobo-Dioulasso. BP 1091 Bobo- \\ Dioulasso, Burkina Faso. \\ ${ }^{2}$ Institut de l'Environnement et de Recherches Agricoles (INERA). 01 BP 476 Ouagadougou 01, Burkina Faso. \\ *Auteur correspondant ; E-mail : issakasenou@gmail.com; Tél : +226-76434555 Fax : +226-20982577
}

\section{RESUME}

La phytorémediation est une technique alternative d'extraction des métaux lourds dans les sols contaminés. La capacité de Vetiveria nigritana à accumuler le cadmium $(\mathrm{Cd})$, cuivre $(\mathrm{Cu})$, plomb $(\mathrm{Pb})$ et zinc (Zn) a été étudiée dans les sols ferrugineux tropicaux et les sols vertiques du Burkina Faso. L'étude a été conduite sous une ombrière et le taux de survie, l'accumulation des métaux lourds et la croissance de Vetiveria nigritana aux différentes concentrations de $\mathrm{Cd}, \mathrm{Cu}, \mathrm{Pb}$ et $\mathrm{Zn}$ ont été déterminés. Les sols ont été contaminés aux métaux lourds par application de déchets urbains solides aux doses de 0, 10, 15 et 20 tonnes/ha. Les résultats ont montré un potentiel de Vetiveria nigritana à accumuler les métaux lourds. Les feuilles ont exporté 4, 5, 9 et 14 fois plus de $\mathrm{Cd}, \mathrm{Zn}, \mathrm{Cu}$ et $\mathrm{Pb}$ que les racines. Les plantes cultivées dans les sols ferrugineux ont exporté des quantités de métaux lourds supérieures $(0,91 \mathrm{mg}$ de $\mathrm{Cd}, 1,75 \mathrm{mg}$ de $\mathrm{Cu}, 2,30 \mathrm{mg}$ de $\mathrm{Pb}$ et 17,69 mg de $\mathrm{Zn}$ ) comparativement aux plantes cultivées dans les vertiques $(0,57 \mathrm{mg}$ de $\mathrm{Cd}, 0,56 \mathrm{mg}$ de $\mathrm{Cu}, 0,25 \mathrm{mg}$ de $\mathrm{Pb}$ et 6,61 mg de $\mathrm{Zn}$ ). Les biomasses sèches des feuilles et des racines ont augmenté avec les quantités de déchets apportées. Elles sont de 13,84 et 26,99 g de feuilles respectivement avec les applications de déchets aux doses de 0 et 20 tonnes/ha, et 8,88 et $32,99 \mathrm{~g}$ de racines avec les doses de 0 et 20 tonnes/ha. L'apport de déchets urbains solides a augmenté la performance des plantes tant du point de vue de la croissance en hauteur $(87,5 \%)$ que du nombre de talles $(84,7 \%)$.

(C) 2012 International Formulae Group. All rights reserved.

Mots clés : Vetiveria nigritana, déchets urbains solides, phytoextraction, métaux lourds, phytoremediation.

\section{INTRODUCTION}

Les sols agricoles sont de plus en plus contaminés par les métaux lourds particulièrement dans les pays tropicaux (Thi, 2009). Les métaux lourds tels que le plomb $(\mathrm{Pb})$, le cadmium $(\mathrm{Cd})$, le cuivre $(\mathrm{Cu})$ et le zinc $(\mathrm{Zn})$ ne sont pas biodégradables et donc peuvent persister dans le sol pendant de longues périodes. De plus, ces métaux sont continuellement rajoutés dans les sols par diverses activités anthropogéniques telles que les industrielles avec les industries métallurgiques et de tanneries ou agricoles avec l'application des déchets urbains solides 
et les boues d'épuration. $\mathrm{Le} \mathrm{Zn}, \mathrm{Cu}$ et $\mathrm{Pb}$ sont importants car ils peuvent être toxiques pour les végétaux et les animaux y compris l'homme. Le souci de Cd découle de son éventuelle entrée dans la chaîne alimentaire, et les troubles rénaux sont les premiers signes biochimiques de la toxicité du $\mathrm{Cd}$. Si ces métaux sont lixiviés dans le sol, ils peuvent polluer les réserves d'eaux souterraines, en particulier dans les zones à nappe phréatique élevée.

Ces dernières années, le développement de techniques efficaces pour décontaminer les sites pollués est devenu indispensable. L'une d'elles, la phytoremédiation, exploite les propriétés de certaines plantes à accumuler de grandes quantités de métaux lourds (Prabha et al., 2007). La phytoremédiation présente de nombreux avantages. Elle est viable économiquement car on peut exploiter les végétaux produits et récupérer les métaux à haute valeur ajoutée, et elle est compatible avec les politiques de préservation de l'environnement.

Cependant, les espèces de Vetiveria sont pérennes et leur longueur peut atteindre 4 $\mathrm{m}$. Leur système racinaire est massif et complexe. Il existe une douzaine d'espèces poussant dans les zones tropicales mais l'espèce la plus cultivée est Vetiveria nigritana originaire d'Afrique Australe. Les vétivers appartiennent à la famille des Poaceae.

Des études sur le potentiel de phytoremediation de Vetiveria zizanioides sur des sols des sites pollués par les métaux lourds à travers les activités minières ont montré des teneurs en $\mathrm{Cd}$ qui varient entre 0,01 et $0,56 \mathrm{mg} \cdot \mathrm{kg}^{-1}$, entre 1,7 et $7 \mathrm{mg} \cdot \mathrm{kg}^{-1}$ pour le $\mathrm{Cu}$, entre 0,49 et $55,8 \mathrm{mg} \cdot \mathrm{kg}^{-1}$ pour le $\mathrm{Pb}$ et entre 6,8 et $158 \mathrm{mg} \cdot \mathrm{kg}^{-1}$ pour le $\mathrm{Zn}$ (Saida, 2005). En Thaïlande, la capacité de vétiver grass à réhabiliter les sols contaminés par les métaux lourds a été démontrée
(Roongtanakiat, 2009; Nualchavee et al., 2011). En Chine, il y a un développement considérable de la technologie de Vetiveria zizanioides, la plante a été introduite dans la plupart des provinces côtières et au sud de la Chine (Chen et al., 2000). En Philippine, des expériences ont démontré que le vétiver peut survivre dans un milieu extrêmement hostile comme les déchets industriels ou miniers (Nualchavee et al., 2011).

Cependant, l'utilisation de Vetiveria nigritana en phytoremediation n'est pas largement reconnue en raison de l'absence de travaux détaillés sur sa capacité à absorber les contaminants et l'application pratique sur le terrain.

L'objectif de la présente étude était de déterminer la capacité de Vetiveria nigritana à accumuler le $\mathrm{Cd}, \mathrm{Cu}, \mathrm{Pb}$ et $\mathrm{Zn}$. Les hypothèses de travail étaient: (1) Vetiveria nigritana accumule les métaux lourds tels que le $\mathrm{Cd}, \mathrm{Cu}, \mathrm{Pb}$ et $\mathrm{Zn}$; (2) la quantité de métal exportée est fonction de l'état de pollution du sol; (3) la quantité de métal exportée est fonction du type de sol.

\section{MATÉRIEL ET MÉTHODES Sites d'étude}

Les échantillons de sol ont été prélevés en novembre 2010, à la profondeur de 0-20 $\mathrm{cm}$ dans des champs non cultivés des villages

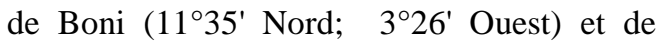
Dossi ( $3^{\circ} 17^{\prime}-3^{\circ} 30^{\prime}$ Ouest; $1^{\circ} 22^{\prime}-11^{\circ} 30^{\prime}$ Nord). Les sols ont été ensuite transportés à l'Université Polytechnique de Bobo-Dioulasso ( $4^{\circ} 10^{\prime}-4^{\circ} 30^{\prime}$ ' Ouest; $11^{\circ}-12$ Nord) au Burkina Faso où l'expérimentation en serre à été conduite (Figure 1). Les sols prélevés à Boni et à Dossi sont respectivement de type ferrugineux tropical lessivé et vertique.

Le climat dans les sites de prélèvement de l'expérimentation est de type sudsoudanien avec une pluviométrie annuelle comprise entre 900 et $1200 \mathrm{~mm}$. 


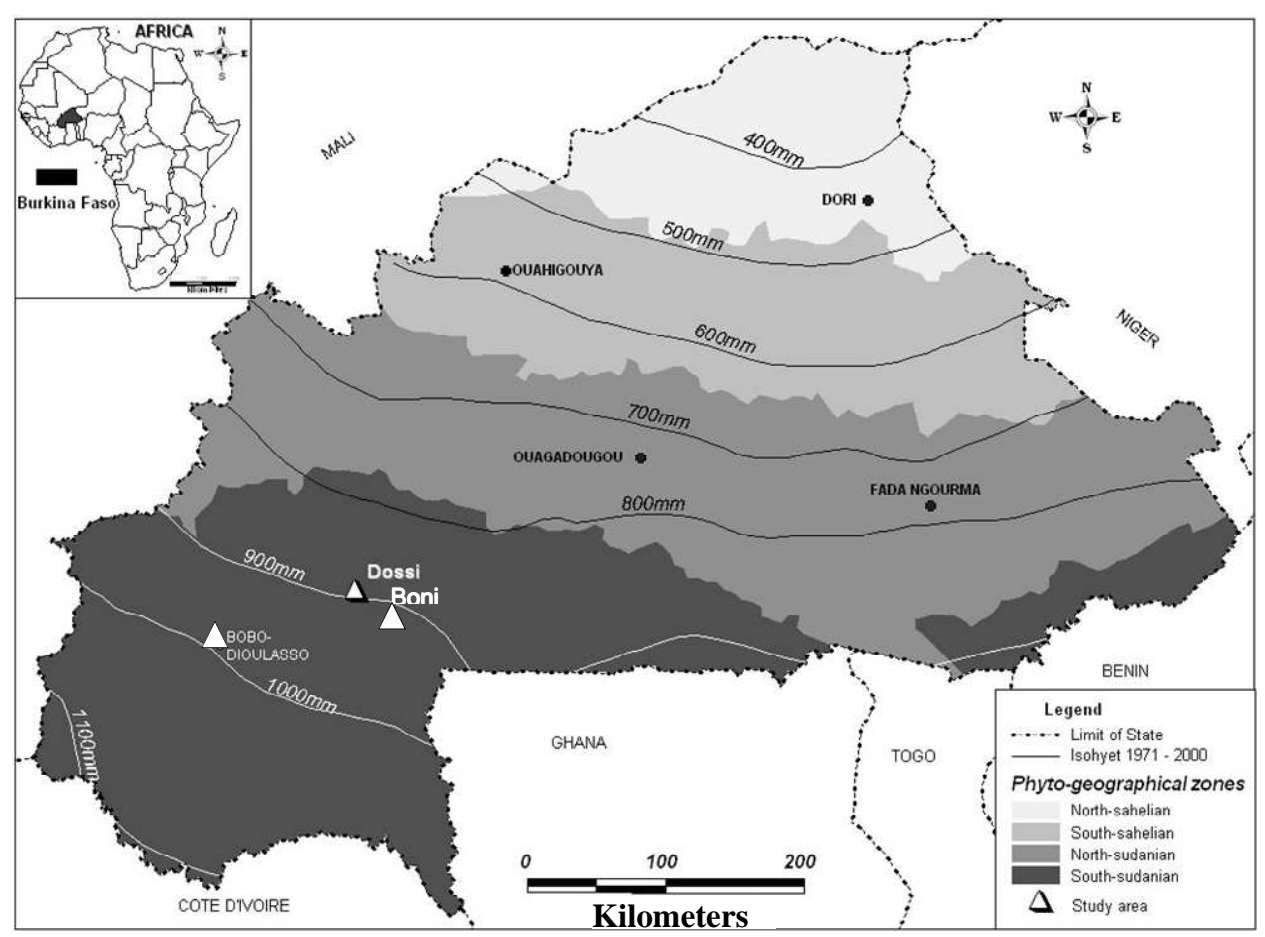

Figure 1: Localisation des sites d'étude.

\section{Plante test}

Le vétiver (Vetiveria nigritana) a été utilisé comme plant test. Il provient d'une pépinière mise en place deux mois avant l'expérimentation. Les plants de vétiver présentant le même développement morphologique ont été sélectionnés et coupés à une hauteur de $40 \mathrm{~cm}$ et la longueur des racines a été réduite à $8 \mathrm{~cm}$.

\section{Dispositif expérimental}

Les seaux en plastique (diamètre intérieur $30 \mathrm{~cm}$ et profondeur $28 \mathrm{~cm}$ ) contenant $10 \mathrm{~kg}$ de terre ont été placés sous une ombrière. Les déchets urbains triés ont été utilisés dans notre expérimentation comme source de pollution en métaux lourds; ils ont été apportés dans les seaux et mélangés intimement au sol. Les plants ont été ensuite repiqués dans les pots. Le dispositif expérimental utilisé est un bloc complètement randomisé avec 4 traitements et répété 3 fois. Pour chaque traitement, 4 seaux ont été utilisés soit un total de 48 seaux. La moitié (2 seaux par traitement) a été détruite à 3 mois après repiquage pour évaluer la biomasse totale et la deuxième à 7 mois après repiquage.

Les traitements sont : i) T0, sans apport de déchet; ii) T1, apport de 3,4 kg de déchet; iii) $\mathrm{T} 2$, apport de $5,1 \mathrm{~kg}$ de déchet; iv) apport de $6,8 \mathrm{~kg}$ de déchet. En termes de quantité de déchets apportés au champ, les traitements T0, $\mathrm{T} 1, \mathrm{~T} 2$ et $\mathrm{T} 3$ correspondent à $0,10,15$ et 20 $\mathrm{t} / \mathrm{ha}$, respectivement.

Les teneurs initiales en métaux lourds dans les déchets purs et dans les différents substrats (sols + déchets) sont représentées par le Tableau 1.

Durant l'expérimentation, la teneur en eau du sol dans les seaux a été maintenue constante à 
$60 \%$ de la capacité maximale de rétention du sol.

\section{Collecte des données}

Pour chaque traitement, l'évolution de la hauteur des plants et du nombre de talle a été mesurée tous les 10 jours. Pour déterminer les biomasses racinaires et aériennes, les plantes entières ont été récoltées dans 2 seaux par traitement à 3 mois et à 7 mois après repiquage. Ces parties végétales ont été lavées à l'eau distillée et séchées à l'air libre puis à l'étuve à $60^{\circ} \mathrm{C}$ jusqu'à poids constant.

\section{Analyse des sols et des plantes}

Les teneurs en métaux lourds ont été déterminées dans les sols et dans les biomasses aériennes et racinaires du vétiver. Les différents échantillons ont été d'abord minéralisés à chaud avec $\mathrm{HNO}_{3}$, puis les teneurs en $\mathrm{Cd}, \mathrm{Cu}, \mathrm{Pb}$ et $\mathrm{Zn}$ ont été déterminées à l'aide d'un spectrophotomètre de masse (ICP-MS).

Après la récolte des plantes ( 3 et 7 mois après repiquage), le sol dans les seaux est uniformément mélangé avant d'être prélevé. Les sols ont ensuite été séchés à l'ombre, puis tamisés à $2 \mathrm{~mm}$ pour les déterminations des paramètres chimiques.

Pour la détermination en azote $(\mathrm{N})$ total, phosphore $(\mathrm{P})$ total et potassium $(\mathrm{K})$ total, les échantillons de sol ont d'abord été minéralisés à chaud avec un mélange $\mathrm{H}_{2} \mathrm{SO}_{4}$ $\mathrm{Se}-\mathrm{H}_{2} \mathrm{O}_{2}$. Par la suite, les teneurs en $\mathrm{N}$ et $\mathrm{P}$ total sont déterminées dans les minéralisâts à l'aide d'un colorimètre automatique SKALAR (Segmented flow analyser, model SANplus 4000-02, Skalar Hollande). Le potassium total a été déterminé à l'aide d'un photomètre de flamme (JENCONS. PFP 7, Jenway LTD, Felsted, England). Le phosphore assimilable a été déterminé par la méthode Bray I (Bray et al., 1945). Le carbone du sol a été déterminé par la méthode de Walkley et al. (1934). La mesure du $\mathrm{pH}$ du sol a été déterminée après agitation pendant $1 \mathrm{~h}$ de $20 \mathrm{~g}$ d'échantillon de sol dans $50 \mathrm{ml}$ d'eau distillée pour le pHeau et $50 \mathrm{ml}$ d'une solution molaire de $\mathrm{KCl}$ pour le pHKCl. La Capacité d'Echange Cationique (CEC) a été déterminée par la méthode de chlorure d'ammonium par la méthode de Metson.

\section{Analyses statistiques}

Les données collectées ont été soumises à une analyse de variance (ANOVA) en utilisant le modèle linéaire général avec le logiciel Minitab (V. 14) pour Windows (Minitab Inc). La séparation des moyennes a été effectuée par le test de Tukey au seuil de $5 \%$.

\section{RESULTATS}

\section{Le taux de survie et tolérance de Vetiveria nigritana aux métaux lourds}

L'application des déchets urbains a augmenté significativement le taux de survie des plants. Par ailleurs, le taux de survie a augmenté avec la quantité de déchets apportée.

\section{Croissance des plantes}

La croissance des plantes est représentée dans les Figures 2 et 3. Quelque soit le type de sol et la durée de la culture, l'apport de déchets urbains solides a augmenté la performance des plantes tant du point de vue de la croissance que du nombre de talles. Toutefois, le taux de croissance en hauteur est plus élevé en sols ferrugineux tropicaux $(61,9 \%)$ comparé aux sols vertiques $(51,81 \%)$. Les biomasses sèches des feuilles et des racines ont augmenté avec les quantités de déchets apportées. Les biomasses foliaires et racinaires sont plus élevées avec les sols ferrugineux tropicaux $(26,99$ et $32,29 \mathrm{~g}$ respectivement des biomasses foliaires et racinaires) comparativement aux sols vertiques $(19,43$ et $21,18 \mathrm{~g}$ respectivement des biomasses foliaires et racinaires). 
Caractéristiques chimiques des sols des différents traitements

Les caractéristiques chimiques des sols des différents traitements avant le repiquage (0 mois) du vétiver et à la récolte ( 3 mois et 7 mois) sont consignées dans le Tableau 2. La période 0 mois correspond au repiquage.

Les sols ferrugineux utilisés ont un pHeau modérément acide $(6,5)$ tandis que les sols vertiques ont un pHeau modérément basique $(7,1)$. L'apport des déchets solides urbains a augmenté le pHeau de 2 unités et 1 unité respectivement pour les sols ferrugineux et vertiques. Quelque soit le traitement et le type de sol, le pHeau a augmenté avec l'apport de déchet et la durée de la mise en culture.

Les teneurs en éléments nutritifs ont augmenté avec la dose d'application des déchets urbains solides. Par contre, le rapport $\mathrm{C} / \mathrm{N}$ a baissé avec l'application des déchets.

Quelque soit le type de sol et la dose de traitement, la CEC a baissé avec le temps de mise en culture. La CEC des sols vertiques est 2,5 fois plus élevée que celle des sols ferrugineux tropicaux. La CEC diminue avec la dose d'apport de déchets pour les deux types de sol.

\section{Métaux lourds exportés (Bioaccumulation de $\mathrm{Cd}, \mathrm{Cu}, \mathrm{Pb}$ et $\mathrm{Zn}$ )}

Les quantités en $\mathrm{Cd}, \mathrm{Cu}, \mathrm{Pb}$ et $\mathrm{Zn}$ exportées par les racines et les feuilles de vétiver sont consignées dans le Tableau 3. En général, les quantités totales exportées augmentent avec la dose de déchets appliqués et la durée de l'expérimentation. Quelque soit la dose appliquée, la quantité de métaux lourds exportés par les feuilles est supérieure à celle des racines. Les feuilles ont exporté 4,5 , 9 et 14 fois plus de $\mathrm{Cd}, \mathrm{Zn}, \mathrm{Cu}$ et $\mathrm{Pb}$ que les racines. Le $\mathrm{Cd}$ est le métal le moins prélevé par la plante $(0,86 \mathrm{mg})$ tandis que le $\mathrm{Zn}$ est le plus prélevé $(17,69 \mathrm{mg})$ soit 20 fois plus.

Les plantes cultivées dans les sols ferrugineux ont exporté des quantités de métaux lourds supérieures comparativement aux plantes cultivées dans les sols vertiques.

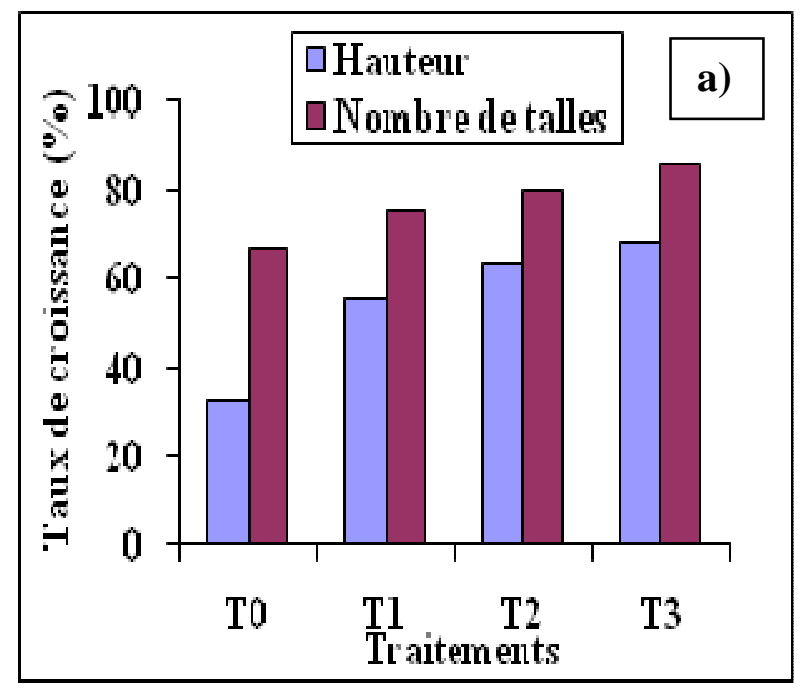



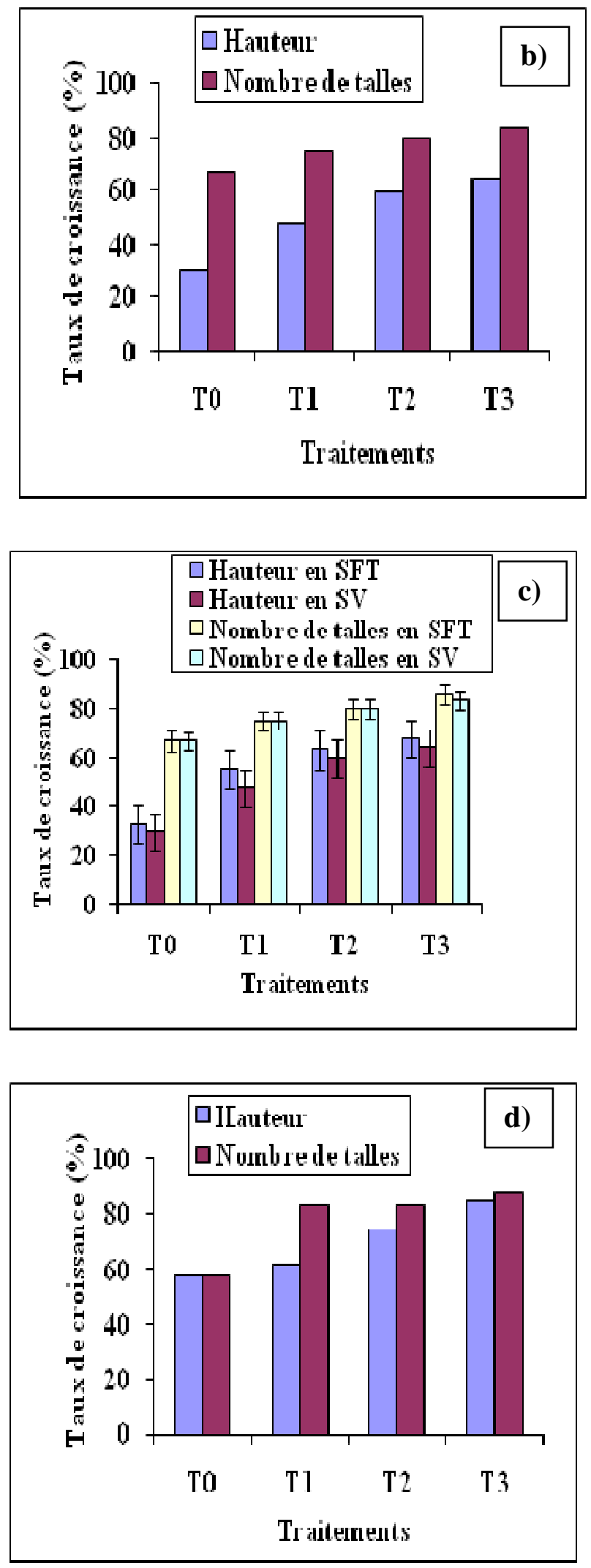

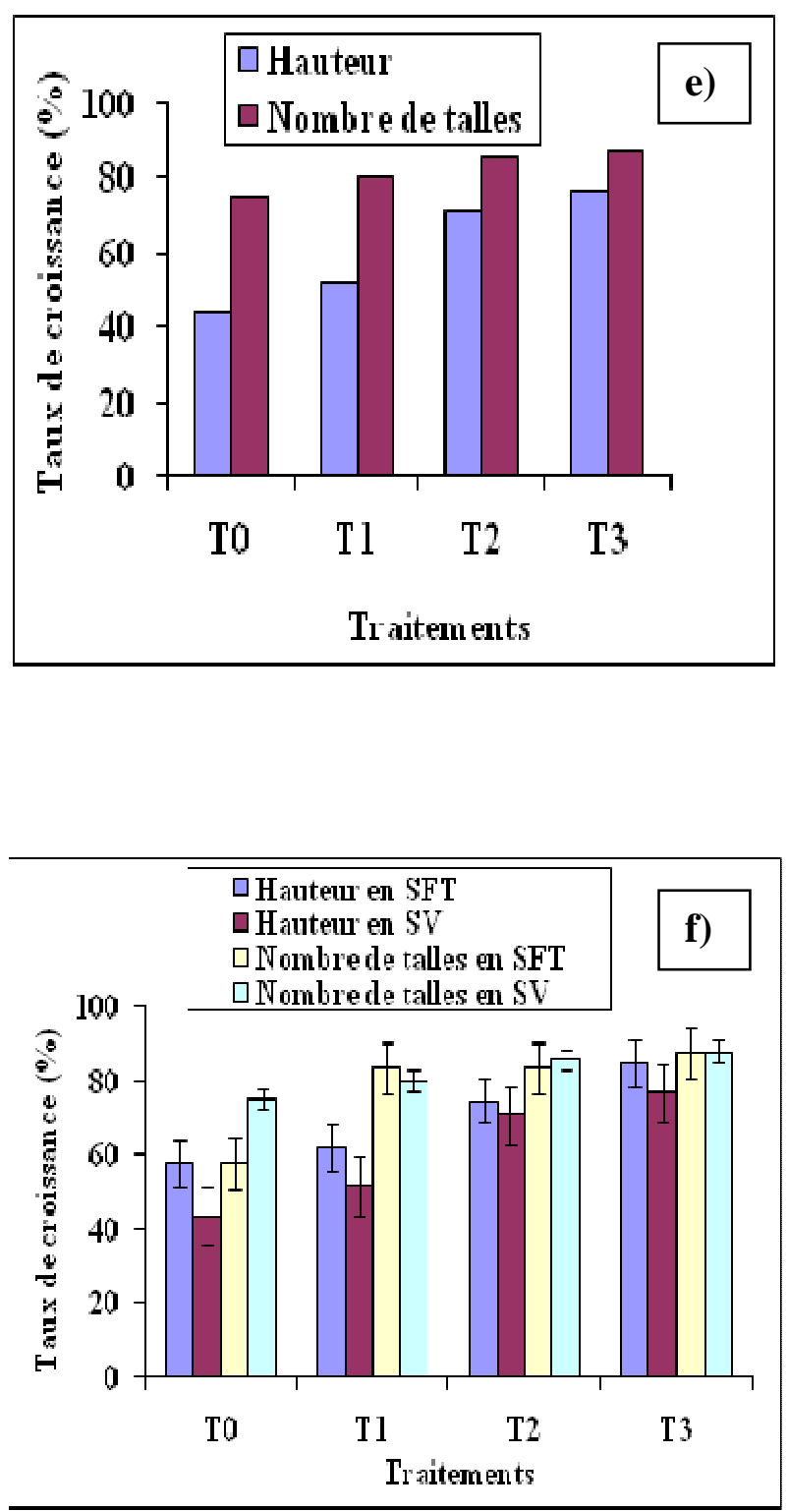

Figure 2 : Taux de croissance en hauteur et du nombre de talles des plantes. a) sur sols ferrugineux tropicaux après 3 mois, b) sur sols vertiques après 3 mois, c) comparaison entre sols ferrugineux tropicaux et sols vertiques après 3 mois, d) sur sols ferrugineux tropicaux après 7 mois, e) sur sols vertiques après 7 mois, f) comparaison entre sols ferrugineux tropicaux et sols vertiques après 7 mois. 

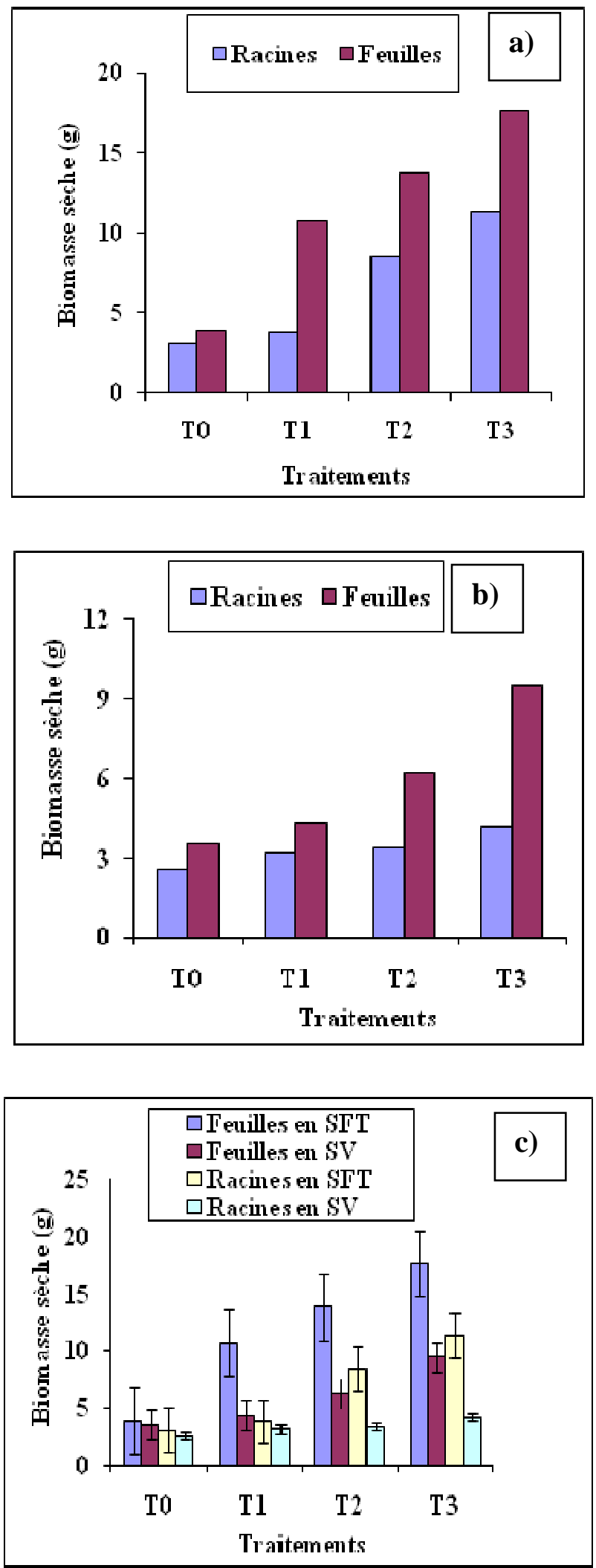

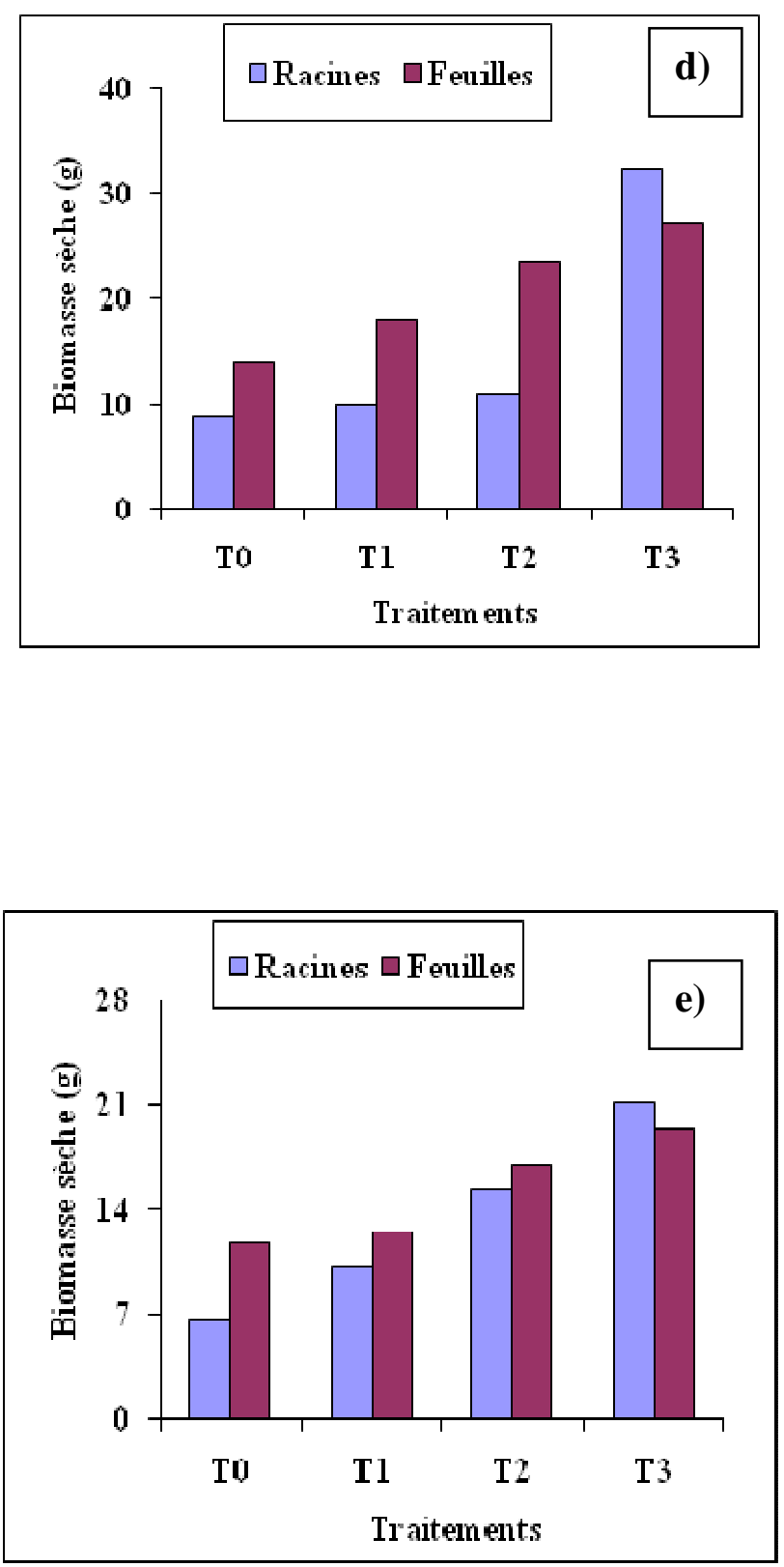


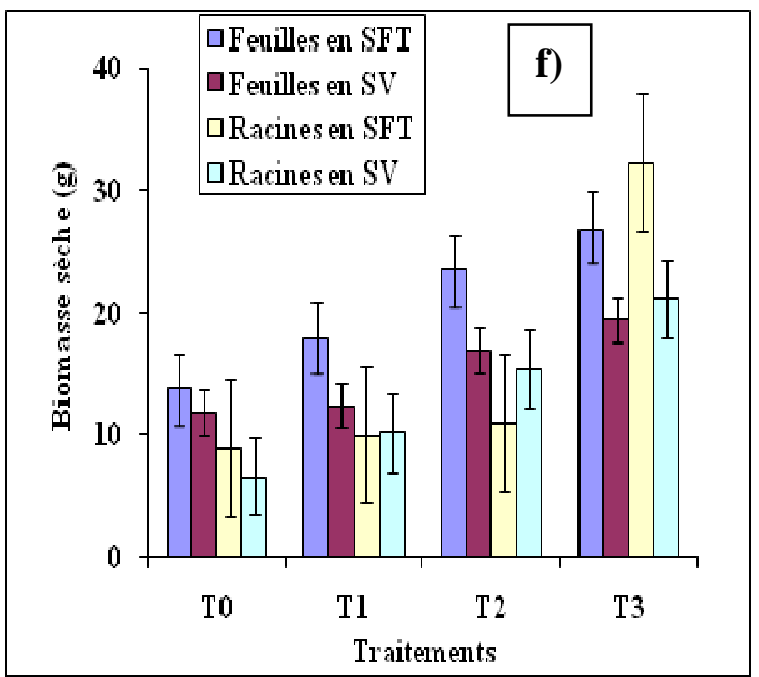

Figure 3 : L'effet des apports de déchets sur les biomasses aérienne et foliaire. a) sur sols ferrugineux tropicaux après 3 mois, b) sur sols vertiques après 3 mois, c) comparaison entre sols ferrugineux tropicaux et sols vertiques après 3 mois, d) sur sols ferrugineux tropicaux après 7 mois, e) sur sols vertiques après 7 mois, f) comparaison entre sols ferrugineux tropicaux et sols vertiques après 7 mois, T0 : sol sans apport de déchets (témoin), T1 : sol avec apport de déchet à la dose de 10 tonnes/ha, T2 : sol avec apport de déchet à la dose de 15 tonnes/ha, T3 : sol avec apport de déchet à la dose de 20 tonnes/ha, SFT : sol ferrugineux tropical, $\mathbf{S V}:$ sol vertique.

Tableau 1: Teneurs initiales en métaux lourds (mg/kg).

\begin{tabular}{lcccc}
\hline \multirow{2}{*}{ Echantillons } & \multicolumn{4}{c}{ Teneurs en métaux lourds $(\mathbf{m g} / \mathbf{k g})$} \\
\cline { 2 - 5 } & $\mathbf{C d}$ & $\mathbf{C u}$ & $\mathbf{P b}$ & $\mathbf{Z n}$ \\
\hline Déchet pur & 1 & 48 & 28 & 313 \\
Sols ferrugineux tropicaux sans apport de déchets & 0,5 & 28 & 2 & 58 \\
Sols ferrugineux tropicaux + 3,4 kg de déchets & 1 & 30 & 4 & 110 \\
Sols ferrugineux tropicaux + 5,1 kg de déchets & 2 & 38 & 26 & 126 \\
Sols ferrugineux tropicaux + 6,8 kg de déchets & 0 & 41 & 20 & 170 \\
Sols vertiques sans apport de déchets & 0 & 87 & 16 & 59 \\
Sols vertiques purs + 3,4 kg de déchets & 0,6 & 71 & 20 & 118 \\
Sols vertiques purs + 5,1 kg de déchets & 1 & 70 & 22 & 160 \\
Sols vertiques purs + 6,8 kg de déchets & 1 & 76 & 25 & 166 \\
\hline \multicolumn{1}{c}{ Cd: cadmium Cu : cuivre Pb : plomb Zn : zinc. } & & & &
\end{tabular}


I. SENOU et al. / Int. J. Biol. Chem. Sci. 6(4): 1437-1452, 2012

Tableau 2: Caractéristiques chimiques des sols des différents traitements.

\begin{tabular}{|c|c|c|c|c|c|c|c|c|c|}
\hline \multirow[b]{2}{*}{ Traitements } & \multicolumn{9}{|c|}{ Paramètres mesurés } \\
\hline & Périodes & pH eau & Carbone (g/kg) & $\mathbf{N}(\mathrm{g} / \mathrm{kg})$ & $\mathbf{C} / \mathbf{N}$ & P-total (mg/kg) & P-ass (mg/kg) & $\mathrm{CEC}$ (C mol/kg) & K-Total (mg/kg) \\
\hline SFT/T0 & 0 mois & 6,47 & 0,081 & 0,006 & 13 & 135,7 & 1,05 & 4,55 & 2245 \\
\hline SFT/T1 & 0 mois & 8,34 & 0,156 & 0,0131 & 12 & 525,4 & 25,49 & 5,55 & 2245 \\
\hline $\mathrm{SFT} / \mathrm{V} / \mathrm{T} 1$ & 3 mois & 8,64 & 0,142 & 0,0149 & 10 & 622 & 50,48 & 4,65 & 2068 \\
\hline $\mathrm{SFT} / \mathrm{V} / \mathrm{T} 1$ & 7 mois & 8,63 & 0,146 & 0,014 & 10 & 609 & 56,99 & 4,90 & 2046 \\
\hline $\mathrm{SFT} / \mathrm{T} 2$ & 0 mois & 8,34 & 0,187 & 0,0168 & 11 & 693,2 & 32,17 & 5,85 & 2134 \\
\hline $\mathrm{SFT} / \mathrm{V} / \mathrm{T} 2$ & 3 mois & 8,64 & 0,165 & 0,0168 & 10 & 672 & 64,97 & 5,00 & 1935 \\
\hline $\mathrm{SFT} / \mathrm{V} / \mathrm{T} 2$ & 7 mois & 8,63 & 0,162 & 0,016 & 10 & 655 & 52,58 & 4,85 & 1935 \\
\hline SFT/T3 & 0 mois & 8,31 & 0,2 & 0,0177 & 11 & 736,2 & 40,32 & 6,45 & 2223 \\
\hline SFT/V/T3 & 3 mois & 8,63 & 0,185 & 0,02 & 9 & 824 & 70,01 & 5,25 & 2046 \\
\hline SFT/V/T3 & 7 mois & 8,56 & 0,158 & 0,016 & 10 & 649 & 60,77 & 5,05 & 1913 \\
\hline SV/T0 & 0 mois & 7,14 & 0,122 & 0,009 & 14 & 152,0 & 1,13 & 15,45 & 1293 \\
\hline SV/T1 & 0 mois & 8,06 & 0,199 & 0,0161 & 12 & 585,5 & 60,14 & 14,8 & 1780 \\
\hline SV/V/T1 & 3 mois & 8,46 & 0,165 & 0,0181 & 9 & 591 & 20,96 & 13,65 & 1647 \\
\hline $\mathrm{SV} / \mathrm{V} / \mathrm{T} 1$ & 7 mois & 8,40 & 0,160 & 0,015 & 11 & 556 & 16,30 & 14,20 & 1625 \\
\hline SV/T2 & 0 mois & 8,16 & 0,217 & 0,0191 & 11 & 752,5 & 67,28 & 13,35 & 1824 \\
\hline $\mathrm{SV} / \mathrm{V} / \mathrm{T} 2$ & 3 mois & 8,47 & 0,189 & 0,019 & 10 & 790 & 31,21 & 11,50 & 1559 \\
\hline SV/V/T2 & 7 mois & 8,47 & 0,19 & 0,018 & 10 & 766 & 30,28 & 11,90 & 1824 \\
\hline SV/T3 & 0 mois & 8,14 & 0,237 & 0,0197 & 12 & 850,3 & 69,38 & 13,6 & 1913 \\
\hline SV/V/T3 & 3 mois & 8,51 & 0,218 & 0,0228 & 10 & 943 & 41,12 & 10,30 & 1824 \\
\hline $\mathrm{SV} / \mathrm{V} / \mathrm{T} 3$ & 7 mois & 8,50 & 0,206 & 0,020 & 10 & 834 & 37,04 & 11,40 & 1692 \\
\hline
\end{tabular}

SFT/T0 : sols ferrugineux tropicaux sans apport de déchet; SFT/T1 : sols ferrugineux tropicaux + déchets à la dose de 10 tonnes/ha; SFT/T2 : sols ferrugineux tropicaux + déchets à la dose de 15 tonnes/ha ; SFT/T3 : sols ferrugineux tropicaux + déchets à la dose de 20 tonnes/ha $;$ SFT/V/T1 : sols ferrugineux tropicaux + déchets à la dose de 10 tonnes/ha + plant de vétiver $;$ SFT/V/T2 : sols ferrugineux tropicaux + déchets à la dose de 15 tonnes/ha + plant de vétiver ; SFT/V/T3 : sols ferrugineux tropicaux + déchets à la dose de 20 tonnes/ha + plant de vétiver ; SV/T0 : sols vertiques sans apport de déchet； SV/T1 : sols vertiques + déchets à la dose de 10 tonnes/ha； SV/T2 : sols vertiques + déchets à la dose de 15 tonnes/ha ; SV/T3 : sols ferrugineux tropicaux + déchets à la dose de 20 tonnes/ha; SV/V/T1 : sols vertiques + déchets à la dose de 10 tonnes/ha + plant de vétiver; SV/V/T2 : sols vertiques + déchets à la dose de 15 tonnes/ha + plant de vétiver; $\mathbf{S V / V / T 3}$ : sols vertiques + déchets à la dose de 20 tonnes/ha + plant de vétiver . 
I. SENOU et al. / Int. J. Biol. Chem. Sci. 6(4): 1437-1452, 2012

Tableau 3 : Quantités de métaux lourds exportés (mg).

\begin{tabular}{|c|c|c|c|c|c|c|c|c|c|}
\hline \multirow{2}{*}{ Partie de la plante } & \multirow{2}{*}{ Traitements } & \multicolumn{2}{|c|}{ Cd } & \multicolumn{2}{|c|}{$\mathbf{C u}$} & \multicolumn{2}{|c|}{$\mathbf{P b}$} & \multicolumn{2}{|c|}{$\mathbf{Z n}$} \\
\hline & & 3 mois & 7 mois & 3 mois & 7 mois & 3 mois & 7 mois & 3mois & 7 mois \\
\hline \multirow{3}{*}{ Racines } & SFT/T1 & 0,05 & 0,10 & 0,12 & 0,20 & 0,03 & 0,04 & 0,64 & 1,11 \\
\hline & $\mathrm{SFT} / \mathrm{T} 2$ & 0,06 & 0,19 & 0,01 & 0,31 & 0,41 & 1,21 & 0,47 & 2,68 \\
\hline & SFT/T3 & 0,36 & 0,46 & 0,32 & 0,88 & 0,68 & 0,50 & 1,73 & 12,14 \\
\hline \multirow{3}{*}{ Feuilles } & $\mathrm{SFT} / \mathrm{T} 1$ & 0,17 & 0,02 & 0,31 & 0,43 & 0,50 & 1,65 & 3,75 & 2,87 \\
\hline & $\mathrm{SFT} / \mathrm{T} 2$ & 0,25 & 0,40 & 0,51 & 0,68 & 1,37 & 0,89 & 2,78 & 5,04 \\
\hline & SFT/T3 & 0,49 & 0,45 & 0,88 & 0,87 & 1,62 & 0,03 & 3,67 & 5,55 \\
\hline \multirow{3}{*}{ Racines } & SV/T1 & 0,04 & 0,07 & 0,12 & 0,03 & 0,13 & 0,26 & 0,63 & 0,61 \\
\hline & SV/T2 & 0,05 & 0,03 & 0,07 & 0,10 & 0,34 & 0,03 & 0,81 & 0,80 \\
\hline & SV/T3 & 0,13 & 0,22 & 0,12 & 0,05 & 0,25 & 0,56 & 0,64 & 3,19 \\
\hline \multirow{3}{*}{ Feuilles } & SV/T1 & 0,06 & 0,16 & 0,13 & 0,45 & 0,37 & 0,10 & 0,54 & 1,59 \\
\hline & $\mathrm{SV} / \mathrm{T} 2$ & 0,12 & 0,03 & 0,13 & 0,53 & 1,03 & 0,48 & 1,44 & 2,02 \\
\hline & SV/T3 & 0,16 & 0,35 & 0,33 & 0,51 & 0,02 & 0,03 & 1,63 & 3,42 \\
\hline $\begin{array}{l}\text { SFT/T1 : sols fer } \\
\text { SFT/T2 : sols fer } \\
\text { SFT/T3 : sols fer } \\
\text { SV/T1 : sols vert } \\
\text { SV/T2 : sols vert } \\
\text { SV/T3 : sols vert }\end{array}$ & $\begin{array}{l}\text { gineux tropicaux }+ \\
\text { gineux tropicaux }+ \\
\text { gineux tropicaux }+ \\
\text { ues }+ \text { déchets à la do } \\
\text { ues }+ \text { déchets à la do } \\
\text { ues }+ \text { déchets à la de }\end{array}$ & $\begin{array}{l}\text { chets à la d } \\
\text { chets à la d } \\
\text { chets à la d } \\
\text { e de } 10 \text { tonr } \\
\text { e de } 15 \text { tonr } \\
\text { e de } 20 \text { tonr }\end{array}$ & $\begin{array}{l}\text { de } 10 \text { ton } \\
\text { de } 15 \text { ton } \\
\text { de } 20 \text { ton } \\
\text { ha } \\
\text { ha } \\
\text { ha }\end{array}$ & & & & & & \\
\hline
\end{tabular}




\section{DISCUSSION}

\section{Le taux de survie et tolérance de Vetiveria nigritana aux métaux lourds}

Le fort taux de survie observé, après 3 et 7 mois de culture, pour les plantes poussant dans des sols contaminés par des déchets urbains et l'absence de dommages confirment la tolérance de Vetiveria nigritana aux métaux lourds dans la limite des doses apportées.

Malgré les fortes teneurs en $\mathrm{Cd}(0,6$ $\mathrm{mg} / \mathrm{kg})$, en $\mathrm{Cu}(87 \mathrm{mg} / \mathrm{kg})$, en $\mathrm{Pb}(28 \mathrm{mg} / \mathrm{kg})$ et en $\mathrm{Zn}(313 \mathrm{mg} / \mathrm{kg})$ observées dans les sols étudiés, la performance de l'espèce étudiée n'a pas été altérée. Nos résultats sont concordants avec ceux de Chen et al. (2000) qui confirment la tolérance de Vetiveria zizanioides à ces métaux. Les travaux de Thy (2009) confirment également la tolérance de Lantana camara (Verbenaceae) à ces métaux. Des résultats similaires ont été trouvés par Santosh et al. (2009) sur le Jatropha curcas lorsque cette plante est développée dans des pots contenant les sols contaminés aux métaux lourds. Ces résultats ont été également confirmés par Lehmann et al. (2004) en comparant deux clones de Calamagrostis epigejos, l'un issu d'un terrain contaminé par du zinc et l'autre issu d'un terrain non pollué, cette espèce présentait une forte tolérance au zinc, mais aussi au plomb et au cadmium, et ceci sans distinction entre les deux clones.

Dans un sol contaminé au $\mathrm{Cu}, \mathrm{Cd}, \mathrm{Pb}$ et $\mathrm{Zn}$, Vetiveria nigritana atteint une biomasse plus élevée que les témoins. Ces résultats peuvent être comparés à ceux d'Epelde et al. (2008) pour la plante hyperaccumulatrice Thlaspi caerulescens qui, lorsqu'elle est exposée à des pollutions au $\mathrm{Cd}$ et au $\mathrm{Zn}$, montre une biomasse supérieure à celle observée chez les témoins.

\section{Croissance des plantes}

Les résultats obtenus ont montré que les apports des métaux lourds à travers les déchets urbains ont augmenté la croissance des plantes. Plusieurs études ont montré que l'amendement organique conduit à la revégétalisation des sols contaminés par des métaux (Ye et al., 2000 ; Ortiz et al., 2006 ; Kumar et al., 2008 ; Juwarkar et al., 2008).

\section{Caractéristiques chimiques des sols et des déchets}

Les résultats indiquent une augmentation modérée du $\mathrm{pH}$ pendant la durée de la mise en culture. Des gammes de $\mathrm{pH}$ proches de la neutralité avant cultures $(6,5$ et 7,1 respectivement en sols ferrugineux tropicaux et vertiques) et basiques après cultures $(8,5$ et 8,1 respectivement en sols ferrugineux tropicaux et vertiques) (Tableau 2) peuvent influer sur la capacité d'adsorption des métaux lourds. Cette augmentation de $\mathrm{pH}$ induit souvent la formation d'espèces précipitées qui peuvent limiter la solubilité et la biodisponibilité de toutes les espèces ioniques (Deneux-Mustin et al., 2003). Cependant, cette augmentation entraîne également la dissolution de matières organiques et la formation consécutive de complexes organométalliques plus solubles (Chaignon, 2001). Les variations de $\mathrm{pH}$ ont donc des conséquences complexes et parfois contraires sur la mobilité des métaux lourds. L'augmentation des teneurs en éléments nutritifs dans les sols au cours du temps pourrait s'expliquer par la minéralisation de la matière organique qui a favorisé la libération des éléments nutritifs. La carence des principaux éléments nutritifs $(\mathrm{N}, \mathrm{P}, \mathrm{K})$ peut affecter l'établissement des plants sur les métaux lourds affectés dans les sols (Cooke et Johnson, 2002).

La CEC diminue avec la dose de déchets et la durée de mise en culture. Ces résultats sont en relation avec la durée de la culture qui a favorisé cette baisse de la CEC. Cette diminution de la CEC est en contradiction avec les résultats rapportés sur des sols ferralitiques (Zombré, 2006); ces résultats ont montré que les substrats organiques doivent maintenir, voire relever le niveau de fertilité. 
Métaux lourds exportés (Bioaccumulation de $\mathrm{Cd}, \mathrm{Cu}, \mathrm{Pb}$ et $\mathrm{Zn}$ )

Les résultats ont montré la capacité de vétiver à accumuler de fortes teneurs de $\mathrm{Cd}$, de $\mathrm{Cu}$, de $\mathrm{Pb}$ et de $\mathrm{Zn}$. Cela se justifie par le fait que cette plante produisant une grande quantité de biomasse (foliaire et racinaire) puisse être stimulée pour accumuler de grandes quantités de métaux lourds.

De nombreux travaux ont fait l'état d'une accumulation de métaux lourds dans les organes des plantes. Les études de Michel (2009) ont montré que lorsque les plants ont subi le traitement en chambre de culture avec $\mathrm{CdCl}_{2}(75 \mu \mathrm{M})$, qu'ils aient été régénérés ou non en présence de $\mathrm{Cd}$, il apparaît des teneurs élevées en $\mathrm{Cd}$ au niveau des feuilles.

Les résultats similaires ont été observés avec Adhikari et al. (2010). En effet, ces travaux ont montré que Typha augustifolia L. (Typhaceae) est capable d'accumuler les fortes concentrations de $\mathrm{Pb}$ dans les racines (1200 mg. $\mathrm{kg}^{-1}$ ) et dans les feuilles (275 mg.kg $\left.{ }^{1}\right)$ tandis que Ipomoea carnea L. (Convolvulaceae) pouvait également accumuler 1500 et $475 \quad \mathrm{mg} \cdot \mathrm{kg}^{-1}$ respectivement dans les racines et dans les feuilles. Les travaux de Thi (2009) ont prouvé que Lantana camara accumule le plomb dans ses racines $\left(3284,08 \mathrm{mg} \cdot \mathrm{kg}^{-1}\right)$ puis celui-ci est transféré vers les parties aériennes $(552,77$ $\left.\mathrm{mg} \cdot \mathrm{kg}^{-1}\right)$ où il est également accumulé en grande quantité. Toutefois, le potentiel de Lantana camara en tant que plante phytoextractrice de $\mathrm{Pb}$ et de $\mathrm{Cd}$ a déjà été prouvé par Diep (2005). Des résultats similaires chez Arabidopsis halleri ont été obtenus avec le $\mathrm{Cd}$ et le $\mathrm{Zn}$ (Zhao et al., 2000). A. halleri avait des teneurs foliaires de $300 \mathrm{mg} \cdot \mathrm{kg}^{-1}$ de $\mathrm{Cd}$ et $20800 \mathrm{mg} \cdot \mathrm{kg}^{-1}$ de $\mathrm{Zn}$, valeurs tout à fait conformes aux références bibliographiques disponibles pour cette espèce (Zhao et al., 2000). Ces teneurs sont conformes à celles reportées par les travaux de Vandecasteele et al. (2008) qui ont montré une augmentation des teneurs de $\mathrm{Cd}$ et de $\mathrm{Zn}$ chez Acer sp., B. pendula et F. excelsior.

Des résultats semblables ont été observés pour le bouleau, l'érable, le tilleul, le saule (Piczak et al., 2003) et le peuplier (Laureysens et al., 2004). Aux Etats-Unis, Alyssum corsicum et Alyssum corsicum sont utilisées pour la phytoextraction du nickel; elles peuvent accumuler plus de 20000 mg.kg${ }^{1}$ de nickel dans les parties aériennes (Chaney et al., 2007).

$\mathrm{La}$ faible teneur en $\mathrm{Pb}$ pourrait se justifier par la faible mobilité de ce métal dans le sol. Ces résultats obtenus sont en accord avec les observations de Orlowska et al. (2002) qui ont montré que le plomb a une faible mobilité dans le sol (moins mobile que le $\mathrm{Zn}$ ). Cela pourrait s'expliquer par le fait que certains métaux comme le $\mathrm{Cd}$ et le $\mathrm{Pb}$ forment dans le sol des complexes organiques qui les rendent indisponible pour les plantes. Les fortes teneurs en $\mathrm{Zn}$ et en $\mathrm{Cu}$ peuvent se justifier par le fait que ces métaux sont des oligo-éléments qui sont naturellement prélevés par les plantes.

\section{Conclusion}

Notre étude a permis de montrer que Vetiveria nigritana est tolérante aux fortes concentrations en métaux lourds. Elle a aussi été capable d'absorber de grandes quantités de $\mathrm{Cd}, \mathrm{Cu}, \mathrm{Pb}$ et $\mathrm{Zn}$ et d'en transférer une majeure partie vers leurs parties aériennes. Cette plante peut donc être envisagée pour décontaminer, à faible coûts, les sols ferrugineux tropicaux et vertiques contaminés par le $\mathrm{Cd}, \mathrm{Cu}, \mathrm{Pb}$ et $\mathrm{Zn}$.

\section{REMERCIEMENTS}

Nos remerciements s'adressent au projet CORUS II $\mathrm{N}^{\circ} 6043$ qui a entièrement financé ces travaux.

\section{REFERENCES}

Adhikari T, Ajay K, Singh MV, Subba AR. 2010. Phytoaccumulation of lead by selected wetland plant species. Soil Sci. Plant Anal., 41: 2623-2632.

Bray RH, Kurtz LT. 1945. Determination of total organic and available forms of phosphorus in soils. Soils Sci., 59: 39-45.

Chaignon V. 2001. Biodisponibilité du cuivre dans la rhizosphère de différentes plantes 
cultivées. Cas de sols viticoles contaminés par des fongicides. Thèse de doctorat, Université d'Aix-Marseille, Marseille, p. 103.

Chaney RL, Angle JS, Broadhurst CL, Peters CA, Tappero RV, Sparks DL. 2007. Improved understanding of hyperaccumulation yields commercial phytoextraction and phytomining technologies. J. Environ. Qual., 36: 14291443.

Chen HM, Zheng CR, Tu C, Shen ZG. 2000. Chemical methods and phytoremadiation of soil contaminated with heavy metals. Chemosphere, 41: 229-234.

Cooke JA, Johnson MS. 2002. Ecological restoration of land with particular reference to the mining of metals and industrial minerals: a review of theory and practice. Environmental Review, 10: 41-71.

Deneux-Mustin S, Roussel-Debet S, Mustin C, Henner P, Munier-Lamy C, Colle C, Berthelin J, Garnier-Laplace J, Leyval C. 2003. Mobilité et Transfert Racinaire des Eléments en Traces : Influence des Micro-organismes du Sol. TEC. DOC: Paris.

Diep MH. 2005. Study of some plants that have a potential of the accumulation $\mathrm{Pb}$ and $\mathrm{Cd}$ from polluted soils in Vietnam. Biotechnology Review, 3: 503-508.

Epelde L, Becerril JM, Hernandez-Allica J, Barrutia O, Garbisu C. 2008. Functional diversity as indicator of the recovery of soil health derived from Thlaspi caerulescens growth and metal phytoextraction. Appl. Soil Ecol., 39: 299-310.

Juwarkar AA, Yadav SK, Kumar GP, Singh SK. 2008. Effect of biosludge and biofertilizer amendment on growth of Jatropha curcas in heavy metal contaminated soils. Environ. Monit. Assess., 145: 7-15.

Kumar GP, Yadav SK, Thawale PR, Singh SK, Juwarkar AA. 2008. Growth of Jatropha curcas on heavy metal contaminated soil amended with industrial wastes and Azotobacter - a greenhouse study. Bioresource Technology, 99: 2078-2082.

Laureysens I, Blust R, Temmerman DL, Lemmens C, Ceulemans R. 2004. Clonal variation in heavy metal accumulation and biomass production in a poplar coppice culture: I. Seasonal variation in leaf, wood and bark concentrations. Environ. Pollut., 131: 485-494.

Lehmann C, Rebele F. 2004. Evaluation of heavy metal tolerance in Calamagrostis epigejos and Elymus repens revealed copper tolerance in a copper smelter population of C. epigejos. Environ. Exp. Bot., 51: 199-213.

Michel A. 2009. Action du cadmium sur les plants de moutarde indienne (Brassica juncea (L.) Czern) néoformés à partir de couches cellulaires minces et issus de semis. Analyses physiologiques et rôle des polyamines. Thèse de doctorat, Université de Bretagne Occidentale, Bretagne, p. 100.

Nualchavee R, Sarattana S. 2011. Phytoextraction of Zinc, Cadmium and Lead from Contaminated Soil by Vetiveria zizanioides. J. Nat. Sci., 45(4): $603-612$.

Orłowska E, Zubek SZ, Jurkiewicz A, SzarekŁukaszewska G, Turnau K. 2002. Influence of restoration on arbuscular mycorrhiza of Biscutella laevigata L. (Brassicaceae) and Plantago lanceolata L. (Plantaginaceae) from calamine spoil mounds. Mycorrhiza, 12: 153-160.

Ortiz O, Alcaniz JM. 2006. Bioaccumulation of heavy metals in Dactylis glomerata L. growing in a calcareous soil amended with sewage sludge. Bioresource Technology, 97: 545-552.

Piczak K, Lesniewicz A, Zyrnicki W. 2003. Metal concentrations in deciduous tree leaves from urban areas in Poland. Environ. Monit. Assess., 86: 273-287.

Prabha K, Loretta YL. 2007. Phytoremediation Technology: Hyperaccumulation Metals in Plants. Water Air Soil Poll., 184: 105-126. 
Roongtanakiat N. 2009. Vetiver Phytoremediation for Heavy Metal Decontamination. Tech. Bull., $\mathrm{N}^{\circ}$. 2009/1, ORDPB, Bangkok, Thailand.

Saida N. 2005. Essais de phytoremédiation par le Vétiver grass (Vetiveria zizanoides). http://www.vetiver.com. Consulté le 22 Janvier 2009.

Santosh KY, Asha AJG, Phani K, Prashant RT, Sanjeev KS, Tapan C. 2009. Bioaccumulation and phyto-translocation of arsenic, chromium and zinc by Jatropha curcas L.: Impact of dairy sludge and biofertilizer. Bioresource Technology, 100: 4616-4622.

Thi MDH. 2009. Impacts des métaux lourds sur l'interaction plante/ver de terre/microflore tellurique. Thèse de doctorat, Université Paris Est, Paris, p. 145.

Vandecasteele B, Samyn J, De VB, Muys B. 2008. Effect of tree species choice and mineral capping in a woodland phytostabilisation system: A case-study for calcareous dredged sediment landfills with an oxidised topsoil. Ecol. Eng., 32: 263-273.

Walkley A, Black A. 1934. An examination of the Degtjareff method for determining soil organic matter and a proposed modification of the chromic acid titration method. Soil Sci., 37: 29-38.

Ye ZH, Wong JWC, Wong MH. 2000. Revegetation of $\mathrm{Pb} / \mathrm{Zn}$ mine tailings, Guangdong Province, China. Restor. Ecol., 8: 87-92.

Zhao FJ, Lombi E, Breedon T, McGrath SP. 2000. Zinc hyperaccumulation and cellular distribution in Arabidopsis halleri. Plant Cell Environ., 23: 507-514.

Zombré PN. 2006. Variation de l'activité biologique dans les zipella (sols nus) en zone subsahélienne du Burkina Faso et impact de la technique du zaï (techniques des poquets). Biotechnol. Agron. Soc. Environ., 10(2): 139-148. 